\title{
Barbara Owen, James Wells and Joycelyn Pollock, In Search of Safety: Confronting Inequality in Women's Imprisonment, University of California Press: Oakland, CA, 2017; 280 pp.: ISBN: 9780520288720
}

Reviewed by DARJA TADIČ ${ }^{1}$

In Search of Safety takes a close look at the sources of violence and conflicts in women's prisons. As the reader will probably note, the authors distance themselves from the individual pathology approach to explaining interpersonal conflicts and violence, focusing instead on unravelling the structural inequalities that shape conflict contexts in prisons. This approach reflects the authors' sociological perspective, as they tackle the topic by using gender, intersectionality, structural inequalities, life trajectories and capital as central conceptual investigative tools.

The book has several overlapping

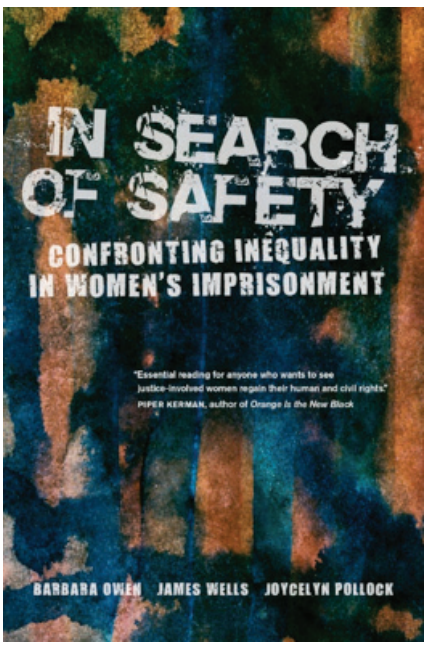
goals. In addition to witnessing and documenting women's experiences with imprisonment, it offers a new analysis of the contemporary prison by reframing the question of trouble and violence as a further expression of broader societal inequalities. The reader should notice the absence of the increasingly present (in criminology, social sciences and public debates, as well) "security" and "dangerous criminal others" discourse. Rather than searching for dangerous prisoners, the book reveals broader violent and unsafe social structures that create interpersonal violence.

The authors examine how intersectional inequalities and cumulative disadvantages are the root of prison conflict and violence. Women must negotiate these inequities by developing forms of prison capital (social, human, cultural, emotional and economic) to ensure their safety while inside. The authors also analyse how conflict and subsequent violence result from the gendered context of substandard prison conditions, inequalities of capital among those imprisoned, and relationships with correctional staff.

1 Faculty of Education, University of Ljubljana, Slovenia; darja.tadic@pef.uni-lj.si. 
Several past research findings are integrated in the book, with the authors drawing on data they had collected over the course of ten years in three studies of women's prisons and jails. In these overlapping studies, they interviewed over 150 imprisoned women and dozens of staff members using the mixed methods approach. Their research was undertaken through open-ended focus groups, asking participants to describe experiences and perceptions of violence and safety in prisons. Through these interviews, the authors also developed a survey instrument to measure women's perception of safety and violence, validating the questionnaire with over four thousand women around the country.

In the first chapter (Intersectional Inequality and Women's Imprisonment), the authors open the book by presenting the central concepts used in their research. They emphasise the fact that women's multiple and cumulative disadvantages and everyday lives in the community condition their pathways to prison. As well as focusing on gender-specific experiences, they also use an intersectional approach by taking into account the importance of the interconnected identities and statuses of individuals and groups in relation to crime and criminality. The reader is warned of the importance of understanding that women's prison experience is also shaped by their "prisoner" status.

The second chapter (Pathways and Intersecting Inequality) describes women's pathways to incarceration, focusing on the impact of intersectional inequalities in women's lives in the free world. Their lives prior to prison are often shaped by their gendered life circumstances and roles, such as the predominance of the caregiving role with regard to children and housing, experiences of victimisation and abuse, unequal employment opportunities, and so on. Before prison, most of the women lived unsafe lives. For many, threats to their physical, sexual, emotional and psychological safety can be found in their homes, neighbourhoods and, often, in their relationships. Intersectional inequality and oppression are significant contributors to women's criminal behaviour.

Chapter 3 (Prison Community, Prison Conditions) examines prison conditions and elements of the prison community that contribute to women's fears over safety while confined. Conditions of material scarcity, substandard living quarters, and few programme and treatment resources are exacerbated by the crowding that characterises contemporary correction institutions. Women's attempts to neutralise, escape or protect can take many forms: bullying another inmate, forming inmate families, withdrawing from certain activities, and other behaviours. In the absence of alternatives, and with the reality of living in a climate of fear, these behaviours offer a sense of control and provide psychological and physical relief. 
In chapter 4 (Searching for Safety through Prison Capital), the authors explore how women's prison culture reflects and responds to gendered inequalities. Prison culture mediates these inequities by mapping cultural routes to survival and safety, while at the same time creating the potential for risk and danger. Inequality within prison is expressed in relations between all members of this community. This chapter also outlines the strategies and tactics that women deploy in their search for safety, which is embedded in prison capital. They face unsafe situations by using various forms of their capital. The authors define "doing safety" as a daily effort that shields women from situational and structural violence in the prison community.

Chapter 5 (Inequalities and Contextual Conflict) explores the impact of inequality on women's imprisonment as it creates and sustains conflict. This chapter provides a detailed description of the forms of gendered violence.

Chapter 6 (Intersections of Inequality with Correctional Staff) expands on the consequences of the obvious inequality between correctional worker and prisoner. In relation to staff, imprisoned women develop various strategies in their search for safety, such as cooperating with rules and regulations, treating staff politely and deferentially, and deflecting staff attention.

In the closing chapter (Gendered Human Rights and the Search of Safety), the authors suggest that women's prisons, because they are unsafe, have become the site of state-sponsored suffering in the reproduction and reinforcement of multiple forms of inequality through the gendered harm of imprisonment. They argue that women's prisons harm women and their life chances in unnecessary ways, concluding: "There is no doubt that all imprisoned people suffer by confinement. We have determined, however, that the overt gender discrimination in the wider society and within the prison adds another punishing layer to the gendered cumulative disadvantage faced by justice-involved women."

The reader interested in research methodologies will probably be inspired by some distinctive methodology steps and specifics that shaped the data collection process in the studies that represent the basis of the book, such as the unique focus group methodology that was developed. The focus groups' questions were developed through a multistage process. Initial questions were pretested in four preliminary prisoners' groups and revised and modified in team discussion with imprisoned women, thus giving them an active role in the process of developing research tools. The imprisoned women also alerted the researchers to the fact that it is not possible to investigate violence and conflict in focus group meetings lasting just an hour or two. They advised the researchers that the topic itself demands the creation of a safe conversation environment, 
which takes time. The authors are aware that the core and the essential part of the findings came about precisely in these prolonged phases of focus group meetings, which were suggested and encouraged by the imprisoned women themselves.

This kind of research approach demonstrated the authors' perception of the imprisoned women as active co-creators in the research process. The research is based on the active participation of the research group (imprisoned women), acknowledging the group as the source of the knowledge and an important subject (rather than just an object) of investigation. Contemporary criminology often does exactly the opposite: it distances itself from the imprisoned (by collecting data from the staff instead) and in so doing neglects the central role that imprisoned people's voices have precisely in the development of prison criminology (e.g., Clemmer, Becker, Gresham and Sykes in the USA, Cohen in England, Mathiesen in Scandinavia, and Foucault in France). The importance of putting the imprisoned person in the role of informer is one of Wacquant's central methodology warnings, as well. Owen, Wells and Pollock's work is therefore an all the more significant contribution to contemporary criminology. It seems that they were fully aware of the difficulty and complexity of prison research.

At the same time, the inclusion of the voices of the imprisoned is all the more important when investigating women's prisons. Only by giving a voice to imprisoned women can the researcher avoid constructing typical (and harmful - a critical mind would say violent) narratives about women in prisons as being passive, conforming and obedient. The authors have enabled them to enforce their right to present and explain their own situation in a first-hand way, giving them the power, which will definitely be an advantage in their future search for safety. 